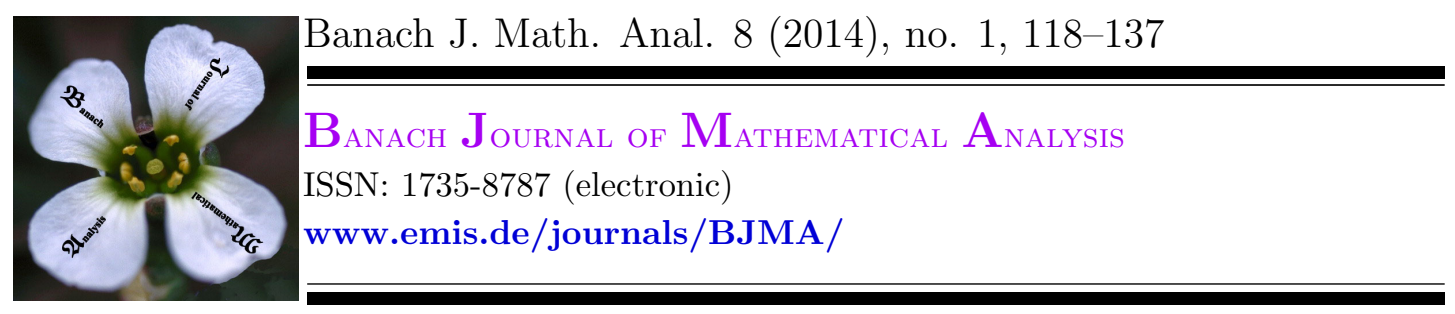

\title{
NEW ESTIMATE OF ESSENTIAL NORM OF COMPOSITION FOLLOWED BY DIFFERENTIATION BETWEEN BLOCH-TYPE SPACES
}

\author{
YU-XIA LIANG AND ZE-HUA ZHOU* \\ Communicated by L. Castro
}

\begin{abstract}
We give a new characterization for the boundedness of composition operator followed by differentiation operator acting on Bloch-type spaces and calculate its essential norm in terms of the $n$-th power of the induced analytic self-map on the unit disk. From which some sufficient and necessary conditions of compactness of the operator follow immediately.
\end{abstract}

\section{INTRODUCTION AND PRELIMINARIES}

The essential norm of a continuous linear operator $T$ is the distance from the operator $T$ to compact operators, that is $\|T\|_{e}=\inf \{\|T-K\|: K$ is compact $\}$. Notice that $\|T\|_{e}=0$ if and only if $T$ is compact, so estimate on $\|T\|_{e}$ will lead to condition for the operator $T$ to be compact.

Let $H(\mathbb{D})$ be the space of all holomorphic functions on $\mathbb{D}$ and $S(\mathbb{D})$ the collection of all holomorphic self-maps on $\mathbb{D}$, where $\mathbb{D}$ is the unit disk in the complex plane $\mathbb{C}$.

For $0<\alpha<\infty$, a holomorphic function $f$ is said to be in the Bloch-type space $\mathcal{B}^{\alpha}$, or $\alpha$-Bloch space, if

$$
\|f\|_{\alpha}=\sup _{z \in \mathbb{D}}\left(1-|z|^{2}\right)^{\alpha}\left|f^{\prime}(z)\right|<\infty .
$$

Date: Received: 4 March 2013; Accepted: 16 May 2013.

* Corresponding author.

2010 Mathematics Subject Classification. Primary 47B38; Secondary 26A24, 32H02, 47B33.

Key words and phrases. Bloch-type space, essential norm, differentiation, composition operator. 
As we all know, the space $\mathcal{B}^{\alpha}$ is a Banach space under the norm

$$
\|f\|_{\mathcal{B}^{\alpha}}=|f(0)|+\sup _{z \in \mathbb{D}}\left(1-|z|^{2}\right)^{\alpha}\left|f^{\prime}(z)\right|
$$

It is known that when $\alpha=1, \mathcal{B}^{\alpha}=\mathcal{B}$, the classical Bloch space; when $0<$ $\alpha<1, \mathcal{B}^{\alpha}=\operatorname{Lip}_{1-\alpha}$, the analytic Lipschitz space which consists of all $f \in H(\mathbb{D})$ satisfying

$$
|f(z)-f(w)| \leq C|z-w|^{1-\alpha}
$$

for some constant $C>0$ and all $z, w \in \mathbb{D}$; when $\alpha>1, \mathcal{B}^{\alpha}=H_{\alpha-1}^{\infty}$, the weighted Banach space of analytic functions that contains all $f \in H(\mathbb{D})$ satisfying

$$
\sup _{z \in \mathbb{D}}\left(1-|z|^{2}\right)^{\alpha-1}|f(z)|<\infty .
$$

We refer the readers to the book [21] by K.H. Zhu.

For $\varphi \in S(\mathbb{D})$ and $u \in H(\mathbb{D})$, we define a weighted composition operator $u C_{\varphi}$ by

$$
u C_{\varphi}(f)=u \cdot(f \circ \varphi)
$$

for $f \in H(\mathbb{D})$. As for $u \equiv 1$, the weighted composition operator is the usual composition operator, denote by $C_{\varphi}$. When $\varphi$ is the identity mapping $I$, the operator $u C_{I}$ is also called multiplication operator. The recent papers or books $[1$, $3,4,5,9,12,17,18,19,20]$ and the related references therein are good sources for information on much of the developments in the theory of composition operators or weighted composition operators. There are still many unsolved problems, some old and some new, that are the interests of numerous mathematicians studying these operators.

Recently interest has arisen to characterize boundedness and compactness of composition operators $C_{\varphi}$ on Bloch-type spaces in terms of the $n$-th power of the analytic self-map $\varphi$ of the open unit disk $\mathbb{D}$. More clearly, Wulan, Zheng and Zhu [15] obtained a new result about the compactness of the composition operator on the Bloch space in the unit disk.

Theorem A. Let $\varphi \in S(\mathbb{D})$. Then $C_{\varphi}$ is compact on the Bloch space $\mathcal{B}$ if and only if

$$
\lim _{n \rightarrow \infty}\left\|\varphi^{n}\right\|_{\mathcal{B}}=0
$$

where $\varphi^{n}$ means the $n$-th power of $\varphi$.

Following their approach, Zhao [16] obtained a beautiful essential norm formula for the composition operator between Bloch-type spaces in in terms of $\varphi^{n}$, which is stated as follows:

Theorem B. Let $0<\alpha, \beta<\infty$ and $\varphi \in S(\mathbb{D})$. Then the essential norm of the composition operator $C_{\varphi}: \mathcal{B}^{\alpha} \rightarrow \mathcal{B}^{\beta}$ is

$$
\left\|C_{\varphi}\right\|_{e}=\left(\frac{e}{2 \alpha}\right)^{\alpha} \limsup _{n \rightarrow \infty} n^{\alpha-1}\left\|\varphi^{n}\right\|_{\beta} .
$$

In [7] Hyvärinen et al. generalized Zhao's work to composition operators on Bloch-type spaces with general radial weights which is non-increasing and tends to zero toward the boundary of $\mathbb{D}$. However, Hyvärinen et al. do not consider 
weights like $\nu_{\log }(z)=\left(1-|z|^{2}\right) \log \left(\frac{2}{1-|z|^{2}}\right)$ which is used to define the logarithmicBloch space. Composition operators from logarithmic-Bloch spaces to weighted Bloch spaces was studied by Castillo et al. in [2](see also [11]). The essential norm formula for composition operator between Bloch-type spaces when $\alpha \neq 1$, was quickly generalized to weighted composition operator by Manhas and Zhao in [10]; however, they were not able to estimate the essential norm of weighted composition operators on the Bloch space $\mathcal{B}$. Very recently, in [6], Hyvärinen and Lindström solved the open problem. Moreover, they presented a direct method to calculate the essential norm of weighted composition operators acting on all Bloch-type spaces $\mathcal{B}^{\alpha}$ in terms of $u$ and $\varphi^{n}$.

The differentiation operator $D$ is defined as

$$
D f=f^{\prime}, f \in H(\mathbb{D}) \text {. }
$$

It is nature to consider the composition operator followed by differentiation operator $D C_{\varphi}$ defined as follows,

$$
D C_{\varphi} f(z)=f^{\prime}(\varphi(z)) \varphi^{\prime}(z), f \in H(\mathbb{D}) .
$$

As we all know, the composition operator is a typical bounded operator on the classical Bloch space $\mathcal{B}$, while the differentiation operators are typically unbounded on many Banach spaces of holomorphic functions. Thus it is also interesting to give a characterization for the boundedness and essential norm of the composition followed by differentiation operator. There has been some work on composition and differentiation operators between holomorphic spaces, for example, [8, 13]. Recently, Wu and Wulan [14] gave a condition for the compactness of the product of differentiation and composition operator acting on the classical Bloch space $\mathcal{B}$ as follows.

Theorem C. Let $\varphi \in S(\mathbb{D})$. Then $D C_{\varphi}$ is compact on $\mathcal{B}$ if and only if $D C_{\varphi}$ is bounded on $\mathcal{B}$ and

$$
\lim _{n \rightarrow \infty}\left\|D \varphi^{n}\right\|_{\mathcal{B}}=0
$$

Building on the above papers, we will give a new estimates for the essential norm of $D C_{\varphi}: \mathcal{B}^{\alpha} \rightarrow \mathcal{B}^{\beta}$ on the unit disk. The paper is organized as follows. In section 2 , we obtain a new characterization of the boundedness of the composition followed by differentiation $D C_{\varphi}: \mathcal{B}^{\alpha} \rightarrow \mathcal{B}^{\beta}$ in terms of $\varphi^{n}$. In section 3, we calculate the essential norm of $D C_{\varphi}: \mathcal{B}^{\alpha} \rightarrow \mathcal{B}^{\beta}$ for all $\alpha, \beta<\infty$, and as corollaries, we characterize compactness of such operators. Some properties are not easily managed, we need some new methods and calculating technics.

Throughout this paper, $C$ will denote a positive constant, the exact value of which will vary from one appearance to the next. The notations $A \asymp B, A \preceq$ $B, A \succeq B$ mean that there maybe different positive constants $C$ such that $B / C \leq A \leq C B, A \leq C B, C B \leq A$. 
2. The Boundedness of $D C_{\varphi}: \mathcal{B}^{\alpha} \rightarrow \mathcal{B}^{\beta}$

In this paper, we will borrow two integral operators which are mentioned in [10]. Let $u \in H(\mathbb{D})$, then for every $f \in H(\mathbb{D})$, define

$$
I_{u} f(z)=\int_{0}^{z} f^{\prime}(\zeta) u(\zeta) d \zeta, \quad J_{u} f(z)=\int_{0}^{z} f(\zeta) u^{\prime}(\zeta) d \zeta
$$

But in the following, we will use the two notations

$$
I_{\varphi^{\prime}}\left(\varphi^{n}\right)(z)=\int_{0}^{z}\left(\varphi^{n}\right)^{\prime}(\zeta) \varphi^{\prime}(\zeta) d \zeta, \quad J_{\varphi^{\prime}}\left(\varphi^{n-1}\right)(z)=\int_{0}^{z} \varphi^{n-1}(\zeta) \varphi^{\prime \prime}(\zeta) d \zeta .
$$

By an easy calculation, it follows that

$$
\left(I_{\varphi^{\prime}}\left(\varphi^{n}\right)(z)\right)^{\prime}=n \varphi(z)^{n-1}\left(\varphi^{\prime}(z)\right)^{2}
$$

and

$$
\left(J_{\varphi^{\prime}}\left(\varphi^{n-1}\right)(z)\right)^{\prime}=\varphi(z)^{n-1} \varphi^{\prime \prime}(z) .
$$

In this section, we will give a new characterization for the bounded composition followed by differentiation $D C_{\varphi}$ from $\mathcal{B}^{\alpha}$ to $\mathcal{B}^{\beta}$. We first list the following Lemma.

Lemma 2.1. Let $\alpha>0, n \in \mathbb{N}$ and $0 \leq x \leq 1$. Let

$$
H_{n, \alpha}(x)=x^{n-1}\left(1-x^{2}\right)^{\alpha} .
$$

Then $H_{n, \alpha}$ has the following properties:

$$
\max _{0 \leq x \leq 1} H_{n, \alpha}(x)=H_{n, \alpha}\left(r_{n}\right)= \begin{cases}1, & n=1 \\ \left(\frac{2 \alpha}{n-1+2 \alpha}\right)^{\alpha}\left(\frac{n-1}{n-1+2 \alpha}\right)^{(n-1) / 2}, & n>1\end{cases}
$$

Where

$$
r_{n}= \begin{cases}0, & n=1 \\ \left(\frac{n-1}{n-1+2 \alpha}\right)^{1 / 2}, & n>1\end{cases}
$$

(2) For $n \geq 1, H_{n, \alpha}$ is increasing on $\left[0, r_{n}\right]$ and decreasing on $\left[r_{n}, 1\right]$.

(3) For $n \geq 1, H_{n, \alpha}$ is decreasing on $\left[r_{n}, r_{n+1}\right]$, and so

$$
\min _{x \in\left[r_{n}, r_{n+1}\right]} H_{n, \alpha}(x)=H_{n, \alpha}\left(r_{n+1}\right)=\left(\frac{2 \alpha}{n+2 \alpha}\right)^{\alpha}\left(\frac{n}{n+2 \alpha}\right)^{(n-1) / 2} .
$$

Consequently,

$$
\lim _{n \rightarrow \infty} n^{\alpha} \min _{x \in\left[r_{n}, r_{n+1}\right]} H_{n, \alpha}(x)=\left(\frac{2 \alpha}{e}\right)^{\alpha}
$$

The proof for the above lemma uses the important limit $\lim _{x \rightarrow \infty}\left(1-\frac{1}{x}\right)^{x}=\frac{1}{e}$ and some easy computations. We omit the details here.

The next lemma is a well-known characterization for the Bloch-type space on the unit disk. 
Lemma 2.2. For $f \in H(\mathbb{D}), m \in \mathbb{N}$ and $\alpha>0$. Then

$$
f(z) \in \mathcal{B}^{\alpha} \Leftrightarrow\|f\|_{\alpha} \asymp \sup _{z \in \mathbb{D}}\left(1-|z|^{2}\right)^{\alpha+m-1}\left|f^{(m)}(z)\right|<\infty .
$$

Hence, when $f \in \mathcal{B}^{\alpha}$, we have that

$$
\|f\|_{\alpha} \asymp \sup _{z \in \mathbb{D}}(1-|z|)^{\alpha+m-1}\left|f^{(m)}(z)\right|<\infty,
$$

where $f^{(m)}$ denotes the $m$-th order derivative of $f \in H(\mathbb{D})$. In this paper, we only use $m=2$, that is,

$$
\|f\|_{\alpha} \asymp \sup _{z \in \mathbb{D}}(1-|z|)^{\alpha+1}\left|f^{\prime \prime}(z)\right|<\infty .
$$

In [8], the authors have characterized the boundedness and compactness of operator $D C_{\varphi}$ between Bloch-type spaces, by proving the following results:

Theorem D. Let $\alpha, \beta>0$ and $\varphi \in S(\mathbb{D})$. Then the following statements hold:

(a) $D C_{\varphi}: \mathcal{B}^{\alpha} \rightarrow \mathcal{B}^{\beta}$ is bounded if and only if

$$
M_{1}:=\sup _{z \in \mathbb{D}} \frac{\left|\varphi^{\prime}(z)\right|^{2}\left(1-|z|^{2}\right)^{\beta}}{\left(1-|\varphi(z)|^{2}\right)^{\alpha+1}}<\infty \quad \text { and } \quad M_{2}:=\sup _{z \in \mathbb{D}} \frac{\left|\varphi^{\prime \prime}(z)\right|\left(1-|z|^{2}\right)^{\beta}}{\left(1-|\varphi(z)|^{2}\right)^{\alpha}}<\infty
$$

(b) $D C_{\varphi}: \mathcal{B}^{\alpha} \rightarrow \mathcal{B}^{\beta}$ is compact if and only if $D C_{\varphi}: \mathcal{B}^{\alpha} \rightarrow \mathcal{B}^{\beta}$ is bounded,

$$
\lim _{|\varphi(z)| \rightarrow 1} \frac{\left|\varphi^{\prime}(z)\right|^{2}\left(1-|z|^{2}\right)^{\beta}}{\left(1-|\varphi(z)|^{2}\right)^{\alpha+1}}=0 \quad \text { and } \quad \lim _{|\varphi(z)| \rightarrow 1} \frac{\left|\varphi^{\prime \prime}(z)\right|\left(1-|z|^{2}\right)^{\beta}}{\left(1-|\varphi(z)|^{2}\right)^{\alpha}}=0 .
$$

The following main result of this section gives a new characterization for the boundedness of $D C_{\varphi}: \mathcal{B}^{\alpha} \rightarrow \mathcal{B}^{\beta}$.

Theorem 2.3. Let $0<\alpha, \beta<\infty$ and $\varphi \in S(\mathbb{D})$. Then $D C_{\varphi}: \mathcal{B}^{\alpha} \rightarrow \mathcal{B}^{\beta}$ is bounded if and only if

$$
\sup _{n \geq 1} n^{\alpha}\left\|I_{\varphi^{\prime}}\left(\varphi^{n}\right)\right\|_{\beta}<\infty
$$

and

$$
\sup _{n \geq 1} n^{\alpha}\left\|J_{\varphi^{\prime}}\left(\varphi^{n-1}\right)\right\|_{\beta}<\infty
$$

Proof. Necessity.

Suppose that $D C_{\varphi}: \mathcal{B}^{\alpha} \rightarrow \mathcal{B}^{\beta}$ is bounded. By the boundedness and (a) of Theorem D, it follows that

$$
M_{1}<\infty \quad \text { and } \quad M_{2}<\infty .
$$

We first show that (2.9) holds. Indeed,

$$
\sup _{n \geq 1} n^{\alpha}\left\|I_{\varphi^{\prime}}\left(\varphi^{n}\right)\right\|_{\beta}=\sup _{n \geq 1} n^{\alpha} \sup _{z \in \mathbb{D}} n\left(1-|z|^{2}\right)^{\beta}|\varphi(z)|^{n-1}\left|\varphi^{\prime}(z)\right|^{2},
$$


and

$$
\begin{aligned}
& \sup _{z \in \mathbb{D}} n^{\alpha+1}\left(1-|z|^{2}\right)^{\beta}|\varphi(z)|^{n-1}\left|\varphi^{\prime}(z)\right|^{2} \\
= & \sup _{z \in \mathbb{D}} \frac{\left(1-|z|^{2}\right)^{\beta}\left|\varphi^{\prime}(z)\right|^{2}}{\left(1-|\varphi(z)|^{2}\right)^{\alpha+1}} n^{\alpha+1}\left(1-|\varphi(z)|^{2}\right)^{\alpha+1}|\varphi(z)|^{n-1} \\
\leq & M_{1} \sup _{z \in \mathbb{D}} n^{\alpha+1}\left(1-|\varphi(z)|^{2}\right)^{\alpha+1}|\varphi(z)|^{n-1} .
\end{aligned}
$$

By Lemma 1, it follows that, for every positive integer $n \geq 1$,

$$
\sup _{z \in \mathbb{D}} n^{\alpha+1}\left(1-|\varphi(z)|^{2}\right)^{\alpha+1}|\varphi(z)|^{n-1} \leq n^{\alpha+1} H_{n, \alpha+1}\left(r_{n}\right),
$$

where $r_{n}$ is defined in (2.3). It is easy to see that

$$
\begin{aligned}
& \lim _{n \rightarrow \infty} n^{\alpha+1} H_{n, \alpha+1}\left(r_{n}\right) \\
& =\lim _{n \rightarrow \infty} n^{\alpha+1}\left(\frac{2(\alpha+1)}{n-1+2(\alpha+1)}\right)^{\alpha+1}\left(\frac{n-1}{n-1+2(\alpha+1)}\right)^{(n-1) / 2} \\
& =\lim _{n \rightarrow \infty}\left[\frac{2(\alpha+1) n}{n-1+2(\alpha+1)}\right]^{\alpha+1}\left[\left(1-\frac{2(\alpha+1)}{n-1+2(\alpha+1)}\right)^{\frac{n-1+2(\alpha+1)}{2(\alpha+1)}}\right]^{\frac{2(\alpha+1)}{n-1+2(\alpha+1)} \frac{n-1}{2}} \\
& =\left(\frac{2(\alpha+1)}{e}\right)^{\alpha+1} .
\end{aligned}
$$

Hence from (2.13), it follows that there exists a constant $K_{1}>0$, independent of $n$, such that for every $n \geq 1$,

$$
n^{\alpha+1} H_{n, \alpha+1}\left(r_{n}\right) \leq K_{1} \text {. }
$$

Thus from (2.11), (12) and (2.14) we obtain that

$$
\sup _{n \geq 1} n^{\alpha}\left\|I_{\varphi^{\prime}}\left(\varphi^{n}\right)\right\|_{\beta} \leq M_{1} K_{1}<\infty
$$

and so (2.9) is true.

Using the similar argument, we can show that (2.10) holds. In fact,

$$
\sup _{n \geq 1} n^{\alpha}\left\|J_{\varphi^{\prime}}\left(\varphi^{n-1}\right)\right\|_{\beta}=\sup _{n \geq 1} \sup _{z \in \mathbb{D}} n^{\alpha}\left(1-|z|^{2}\right)^{\beta}|\varphi(z)|^{n-1}\left|\varphi^{\prime \prime}(z)\right| .
$$

And

$$
\begin{aligned}
& \sup _{z \in \mathbb{D}} n^{\alpha}\left(1-|z|^{2}\right)^{\beta}|\varphi(z)|^{n-1}\left|\varphi^{\prime \prime}(z)\right| \\
= & \sup _{z \in \mathbb{D}} \frac{\left(1-|z|^{2}\right)^{\beta}\left|\varphi^{\prime \prime}(z)\right|}{\left(1-|\varphi(z)|^{2}\right)^{\alpha}} n^{\alpha}|\varphi(z)|^{n-1}\left(1-|\varphi(z)|^{2}\right)^{\alpha} \\
\leq & M_{2} \sup _{z \in \mathbb{D}} n^{\alpha}\left(1-|\varphi(z)|^{2}\right)^{\alpha}|\varphi(z)|^{n-1} \\
\leq & M_{2} n^{\alpha} H_{n, \alpha}\left(r_{n}\right) \\
= & M_{2} n^{\alpha}\left(\frac{2 \alpha}{n-1+2 \alpha}\right)^{\alpha}\left(\frac{n-1}{n-1+2 \alpha}\right)^{\frac{n-1}{2}} .
\end{aligned}
$$


If we note that $\lim _{n \rightarrow \infty} n^{\alpha}\left(\frac{2 \alpha}{n-1+2 \alpha}\right)^{\alpha}\left(\frac{n-1}{n-1+2 \alpha}\right)^{\frac{n-1}{2}}=\left(\frac{2 \alpha}{e}\right)^{\alpha}$ which can be easily checked, then we know that there exists a constant $K_{2}$ such that for every $n \geq 1$,

$$
n^{\alpha} H_{n, \alpha}\left(r_{n}\right) \leq K_{2}
$$

Therefore

$$
\sup _{n \geq 1} n^{\alpha}\left\|J_{\varphi^{\prime}}\left(\varphi^{n-1}\right)\right\|_{\beta} \leq M_{2} K_{2}<\infty .
$$

Which means that (2.10) holds.

Sufficiency.

Suppose that (2.9) and (2.10) hold. If we choose $n=1$ in (2.9) and (2.10), it is clear that

$$
\left\|I_{\varphi^{\prime}}(\varphi)\right\|_{\beta}=\sup _{z \in \mathbb{D}}\left(1-|z|^{2}\right)^{\beta}\left|\varphi^{\prime}(z)\right|^{2}<\infty
$$

and

$$
\left\|J_{\varphi^{\prime}}(1)\right\|_{\beta}=\sup _{z \in \mathbb{D}}\left(1-|z|^{2}\right)^{\beta}\left|\varphi^{\prime \prime}(z)\right|<\infty .
$$

Firstly, if $\sup _{z \in \mathbb{D}}|\varphi(z)|<1$, then there is a number $r$, with $0<r<1$, such that

$$
\sup _{z \in \mathbb{D}}|\varphi(z)|<r .
$$

In this case, by (2.16), (2.17) and (2.6), it follows that for every $\|f\|_{\mathcal{B}^{\alpha}} \leq 1$,

$$
\begin{aligned}
\left\|D C_{\varphi} f\right\|_{\beta} & =\sup _{z \in \mathbb{D}}\left(1-|z|^{2}\right)^{\beta}\left|f^{\prime \prime}(\varphi(z))\left(\varphi^{\prime}(z)\right)^{2}+f^{\prime}(\varphi(z)) \varphi^{\prime \prime}(z)\right| \\
& \leq \sup _{z \in \mathbb{D}}\left(1-|z|^{2}\right)^{\beta}\left|f^{\prime \prime}(\varphi(z))\left(\varphi^{\prime}(z)\right)^{2}\right|+\sup _{z \in \mathbb{D}}\left(1-|z|^{2}\right)^{\beta}\left|f^{\prime}(\varphi(z)) \varphi^{\prime \prime}(z)\right| \\
& =\sup _{z \in \mathbb{D}} \frac{\left(1-|z|^{2}\right)^{\beta}\left|\left(\varphi^{\prime}(z)\right)^{2}\right|}{\left(1-|\varphi(z)|^{2}\right)^{\alpha+1}}\|f\|_{\alpha}+\sup _{z \in \mathbb{D}} \frac{\left(1-|z|^{2}\right)^{\beta}\left|\varphi^{\prime \prime}(z)\right|}{\left(1-|\varphi(z)|^{2}\right)^{\alpha}}\|f\|_{\alpha} \\
& \leq \sup _{z \in \mathbb{D}} \frac{\left(1-|z|^{2}\right)^{\beta}\left|\left(\varphi^{\prime}(z)\right)^{2}\right|}{\left(1-r^{2}\right)^{\alpha+1}}+\sup _{z \in \mathbb{D}} \frac{\left(1-|z|^{2}\right)^{\beta}\left|\varphi^{\prime \prime}(z)\right|}{\left(1-r^{2}\right)^{\alpha}} \\
& <\infty .
\end{aligned}
$$

From (2.18), we obtain the boundeness of $D C_{\varphi}: \mathcal{B}^{\alpha} \rightarrow \mathcal{B}^{\beta}$.

In the following, we assume that $\sup _{z \in \mathbb{D}}|\varphi(z)|=1$. For any $\|f\|_{\mathcal{B}^{\alpha}} \leq 1$, it is clear that

$$
\begin{aligned}
\left\|D C_{\varphi} f\right\|_{\beta} & =\sup _{z \in \mathbb{D}}\left(1-|z|^{2}\right)^{\beta}\left|f^{\prime \prime}(\varphi(z))\left(\varphi^{\prime}(z)\right)^{2}+f^{\prime}(\varphi(z)) \varphi^{\prime \prime}(z)\right| \\
& =\sup _{z \in \mathbb{D}} \frac{\left(1-|z|^{2}\right)^{\beta}\left|\left(\varphi^{\prime}(z)\right)^{2}\right|}{\left(1-|\varphi(z)|^{2}\right)^{\alpha+1}}\|f\|_{\alpha}+\sup _{z \in \mathbb{D}} \frac{\left(1-|z|^{2}\right)^{\beta}\left|\varphi^{\prime \prime}(z)\right|}{\left(1-|\varphi(z)|^{2}\right)^{\alpha}}\|f\|_{\alpha} \\
& \leq \sup _{z \in \mathbb{D}} \frac{\left(1-|z|^{2}\right)^{\beta}\left|\left(\varphi^{\prime}(z)\right)^{2}\right|}{\left(1-|\varphi(z)|^{2}\right)^{\alpha+1}}+\sup _{z \in \mathbb{D}} \frac{\left(1-|z|^{2}\right)^{\beta}\left|\varphi^{\prime \prime}(z)\right|}{\left(1-|\varphi(z)|^{2}\right)^{\alpha}} \\
& =I_{1}+I_{2} .
\end{aligned}
$$


For any integer $n \geq 1$, let

$$
D_{n}=\left\{z \in \mathbb{D}: r_{n} \leq|\varphi(z)| \leq r_{n+1}\right\}
$$

where $r_{n}$ is given by (2.3). Let $k$ be the the smallest positive integer such that $D_{k} \neq \emptyset$. Since $\sup _{z \in \mathbb{D}}|\varphi(z)|=1, D_{n}$ is not empty for every integer $n \geq k$, and $\mathbb{D}=\bigcup_{n=k}^{\infty} D_{n}$. By Lemma 1 , for every $n \geq k$,

$$
\begin{aligned}
& \min _{z \in D_{n}} n^{\alpha+1}|\varphi(z)|^{n-1}\left(1-|\varphi(z)|^{2}\right)^{\alpha+1} \\
& \geq n^{\alpha+1} H_{n, \alpha+1}\left(r_{n+1}\right),
\end{aligned}
$$

Since

$$
\lim _{n \rightarrow \infty} n^{\alpha+1} H_{n, \alpha+1}\left(r_{n+1}\right)=\left(\frac{2(\alpha+1)}{e}\right)^{\alpha+1}
$$

there is $\delta_{1}>0$ independent of $n$ such that

$$
\min _{z \in D_{n}} n^{\alpha+1}\left(1-|\varphi(z)|^{2}\right)^{\alpha+1}|\varphi(z)|^{n-1} \geq \delta_{1}
$$

Hence

$$
\begin{aligned}
I_{1}: & =\sup _{z \in \mathbb{D}} \frac{\left(1-|z|^{2}\right)^{\beta}\left|\left(\varphi^{\prime}(z)\right)^{2}\right|}{\left(1-|\varphi(z)|^{2}\right)^{\alpha+1}} \\
& =\sup _{z \in \mathbb{D}} \frac{\left(1-|z|^{2}\right)^{\beta}\left|\left(\varphi^{\prime}(z)\right)^{2}\right||\varphi(z)|^{n-1} n^{\alpha+1}}{\left(1-|\varphi(z)|^{2}\right)^{\alpha+1}|\varphi(z)|^{n-1} n^{\alpha+1}} \\
& =\sup _{k \leq n} \sup _{z \in D_{n}} \frac{\left(1-|z|^{2}\right)^{\beta}\left|\left(\varphi^{\prime}(z)\right)^{2}\right||\varphi(z)|^{n-1} n^{\alpha+1}}{\left(1-|\varphi(z)|^{2}\right)^{\alpha+1}|\varphi(z)|^{n-1} n^{\alpha+1}} \\
& \leq \frac{1}{\delta_{1}} \sup _{k \leq n} \sup _{z \in D_{n}}\left(1-|z|^{2}\right)^{\beta}\left|\left(\varphi^{\prime}(z)\right)^{2}\right||\varphi(z)|^{n-1} n^{\alpha+1} \\
& \leq \frac{1}{\delta_{1}} \sup _{n \geq 1} \sup _{z \in \mathbb{D}} n^{\alpha}\left|\varphi^{\prime}(z) \|\left(\varphi^{n}\right)^{\prime}(z)\right|\left(1-|z|^{2}\right)^{\beta} \\
& =\frac{1}{\delta_{1}} \sup _{n \geq 1} n^{\alpha}\left\|I_{\varphi^{\prime}}\left(\varphi^{n}\right)\right\|_{\beta}<\infty .
\end{aligned}
$$

On the other hand, using the similar proof as that of (2.20), we know that there is $\delta_{2}>0$ independent of $n$ such that

$$
\min _{z \in D_{n}} n^{\alpha}\left(1-|\varphi(z)|^{2}\right)^{\alpha}|\varphi(z)|^{n-1} \geq \delta_{2}
$$


At the same time, it is clear that

$$
\begin{aligned}
I_{2}: & =\sup _{z \in \mathbb{D}} \frac{\left(1-|z|^{2}\right)^{\beta}\left|\varphi^{\prime \prime}(z)\right|}{\left(1-|\varphi(z)|^{2}\right)^{\alpha}} \\
& =\sup _{z \in \mathbb{D}} \frac{\left(1-|z|^{2}\right)^{\beta}\left|\varphi^{\prime \prime}(z)\right||\varphi(z)|^{n-1} n^{\alpha}}{\left(1-|\varphi(z)|^{2}\right)^{\alpha}|\varphi(z)|^{n-1} n^{\alpha}} \\
& =\sup _{k \leq n} \sup _{z \in D_{n}} \frac{\left(1-|z|^{2}\right)^{\beta}\left|\varphi^{\prime \prime}(z)\right||\varphi(z)|^{n-1} n^{\alpha}}{\left(1-|\varphi(z)|^{2}\right)^{\alpha}|\varphi(z)|^{n-1} n^{\alpha}} \\
& \leq \frac{1}{\delta_{2}} \sup _{n \geq 1} \sup _{z \in \mathbb{D}}\left(1-|z|^{2}\right)^{\beta}\left|\varphi^{\prime \prime}(z)\right||\varphi(z)|^{n-1} n^{\alpha} \\
& =\frac{1}{\delta_{2}} \sup _{n \geq 1} n^{\alpha}\left\|J_{\varphi^{\prime}}\left(\varphi^{n-1}\right)\right\|_{\beta}<\infty .
\end{aligned}
$$

By (2.19), (2.21) and (2.22), we obtain that

$$
\left\|D C_{\varphi} f\right\|_{\beta} \leq \frac{1}{\delta_{1}} \sup _{n \geq 1} n^{\alpha}\left\|I_{\varphi^{\prime}}\left(\varphi^{n}\right)\right\|_{\beta}+\frac{1}{\delta_{2}} \sup _{n \geq 1} n^{\alpha}\left\|J_{\varphi^{\prime}}\left(\varphi^{n-1}\right)\right\|_{\beta}<\infty .
$$

Then $D C_{\varphi}: \mathcal{B}^{\alpha} \rightarrow \mathcal{B}^{\beta}$ is bounded. This completes the proof.

Let $\alpha=\beta=1$ in the above theorem, we obtain the following corollary

Corollary 2.4. Let $\varphi \in S(\mathbb{D})$. Then $D C_{\varphi}: \mathcal{B} \rightarrow \mathcal{B}$ is bounded if and only if

$$
\sup _{n \geq 1} n\left\|I_{\varphi^{\prime}}\left(\varphi^{n}\right)\right\|_{\mathcal{B}}<\infty
$$

and

$$
\sup _{n \geq 1} n\left\|J_{\varphi^{\prime}}\left(\varphi^{n-1}\right)\right\|_{\mathcal{B}}<\infty
$$

3. The essential nORM OF $D C_{\varphi}: \mathcal{B}^{\alpha} \rightarrow \mathcal{B}^{\beta}$

In this section, we will give an estimate for the essential norm of $D C_{\varphi}: \mathcal{B}^{\alpha} \rightarrow$ $\mathcal{B}^{\beta}$. To simplify the notations, we denote

$$
A:=\left(\frac{e}{2(\alpha+1)}\right)^{\alpha+1} \limsup _{n \rightarrow \infty} n^{\alpha}\left\|I_{\varphi^{\prime}}\left(\varphi^{n}\right)\right\|_{\beta}
$$

and

$$
B:=\left(\frac{e}{2 \alpha}\right)^{\alpha} \limsup _{n \rightarrow \infty} n^{\alpha}\left\|J_{\varphi^{\prime}}\left(\varphi^{n-1}\right)\right\|_{\beta} .
$$

Moreover, for $r \in(0,1)$, let $K_{r} f(z)=f(r z)$. It is obvious that $K_{r}$ is a compact operator on the spaces $\mathcal{B}^{\alpha}$ or $\mathcal{B}_{0}^{\alpha}$ for any $\alpha>0$ and with $\left\|K_{r}\right\| \leq 1$.

Next we list three lemmas, they are respectively corresponding to the three different cases $0<\alpha<1, \alpha=1$, and $\alpha>1$.

Lemma 3.1. [9, Lemma 1] Let $0<\alpha<1$. Then there is a sequence $\left\{\gamma_{k}\right\}$, with $0<\gamma_{k}<1$ tending to 1 , such that the compact operator

$$
L_{n}=\frac{1}{n} \sum_{k=1}^{n} K_{\gamma_{k}}
$$


on $\mathcal{B}_{0}^{\alpha}$ satisfies:

(i) For any $t \in[0,1), \lim _{n \rightarrow \infty} \sup _{\|f\|_{\mathcal{B}^{\alpha} \leq 1} \leq 1|z| \leq t} \sup _{|z|}\left|\left(\left(I-L_{n}\right) f\right)^{\prime}(z)\right|=0$.

(ii) $\lim _{n \rightarrow \infty} \sup _{\|f\|_{\mathcal{B}^{\alpha} \leq 1}} \sup _{z \in \mathbb{D}}\left|\left(I-L_{n}\right) f(z)\right|=0$.

(iii) $\limsup _{n \rightarrow \infty}\left\|I-L_{n}\right\| \leq 1$.

Furthermore, these statements hold as well for the sequence of biadjoints $L_{n}^{* *}$ on $\mathcal{B}^{\alpha}$.

Lemma 3.2. [9, Lemma 2] Let $\alpha=1$. Then there is a sequence $\left\{\gamma_{k}\right\}$, with $0<\gamma_{k}<1$ tending to 1 , such that the compact operator

$$
L_{n}=\frac{1}{n} \sum_{k=1}^{n} K_{\gamma_{k}}
$$

on $\mathcal{B}_{0}$ satisfies:

(i) For any $t \in[0,1), \lim _{n \rightarrow \infty} \sup _{\|f\|_{\mathcal{B}} \leq 1} \sup _{|z| \leq t}\left|\left(\left(I-L_{n}\right) f\right)^{\prime}(z)\right|=0$.

(iia) $\limsup _{n \rightarrow \infty} \sup _{\|f\|_{\mathcal{B}} \leq 1} \sup _{|z|>s}\left|\left(I-L_{n}\right) f(z)\right|\left(\log \frac{1}{1-|z|^{2}}\right)^{-1} \leq 1$, for s sufficiently close to 1 , and

(iib) $\lim _{n \rightarrow \infty} \sup _{\|f\|_{\mathcal{B}} \leq 1} \sup _{|z| \leq s}\left|\left(I-L_{n}\right) f(z)\right|=0$, for the above $s$.

(iii) $\limsup _{n \rightarrow \infty}\left\|I-L_{n}\right\| \leq 1$.

Furthermore, the same is true for the sequence of biadjoints $L_{n}^{* *}$ on $\mathcal{B}$.

Lemma 3.3. [16, Lemma 4.3] Let $\alpha>1$. Then there is a sequence $\left\{\gamma_{k}\right\}$, with $0<\gamma_{k}<1$ tending to 1 , such that the compact operator

$$
L_{n}=\frac{1}{n} \sum_{k=1}^{n} K_{\gamma_{k}}
$$

on $\mathcal{B}_{0}^{\alpha}$ satisfies:

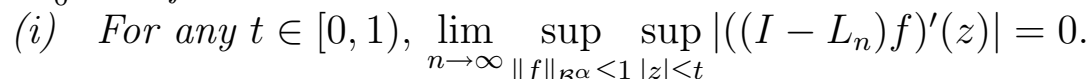

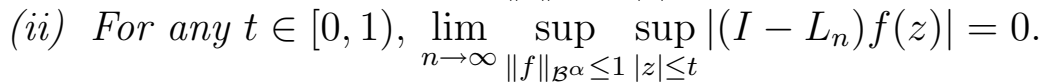

(iii) $\limsup \left\|I-L_{n}\right\| \leq 1$.

Furthermore, these statements hold as well for the sequence of biadjoints $L_{n}^{* *}$ on $\mathcal{B}^{\alpha}$.

The next lemma can be proved in a standard way; see, for example, Proposition 3.11 in [3].

Lemma 3.4. Let $0<\alpha, \beta<\infty$ and $\varphi \in S(\mathbb{D})$. Then $D C_{\varphi}: \mathcal{B}^{\alpha} \rightarrow \mathcal{B}^{\beta}$ is compact if and only if $D C_{\varphi}: \mathcal{B}^{\alpha} \rightarrow \mathcal{B}^{\beta}$ is bounded and for any bounded sequence $\left\{f_{k}\right\}_{k \in \mathbb{N}}$ in $\mathcal{B}^{\alpha}$ which converges to zero uniformly on compact subsets of $\mathbb{D}$, then $\left\|D C_{\varphi} f_{k}\right\|_{\mathcal{B}^{\beta}} \rightarrow 0$ as $k \rightarrow \infty$. 
Theorem 3.5. Let $0<\alpha, \beta<\infty$ and $\varphi \in S(\mathbb{D})$. Suppose that $D C_{\varphi}: \mathcal{B}^{\alpha} \rightarrow \mathcal{B}^{\beta}$ is bounded. Then the estimate for the essential norm of $D C_{\varphi}: \mathcal{B}^{\alpha} \rightarrow \mathcal{B}^{\beta}$ is

$$
\max \left\{\frac{A}{3 \cdot 2^{\alpha+1}}, \quad \frac{B}{2^{\alpha+1}(3 \alpha+4)}\right\} \leq\left\|D C_{\varphi}\right\|_{e} \preceq(A+B) .
$$

Proof. Since $D C_{\varphi}: \mathcal{B}^{\alpha} \rightarrow \mathcal{B}^{\beta}$ is bounded, it follows from Theorem 1 that $A<$ $\infty$ and $B<\infty$. Besides, from the proof of Theorem 1, we know that (2.16) and (2.17) are true.

In the case, $\sup _{z \in \mathbb{D}}|\varphi(z)|<1$. Then there is a number $r \in(0,1)$ such that

$$
\sup _{z \in \mathbb{D}}|\varphi(z)|<r .
$$

In this case, the operator $D C_{\varphi}: \mathcal{B}^{\alpha} \rightarrow \mathcal{B}^{\beta}$ is compact. Indeed, suppose that $\left\{f_{k}\right\}_{k \in \mathbb{N}}$ is a bounded sequence in $\mathcal{B}^{\alpha}$ which converges to zero uniformly on compact subsets of $\mathbb{D}$, and denote $\sup _{k \in \mathbb{N}}\left\|f_{k}\right\|_{\mathcal{B}^{\alpha}} \leq L$. Hence using Cauchy's integral formula, $f_{k}^{\prime}$ and $f_{k}^{\prime \prime}$ converge to zero uniformly on compact subsets of $\mathbb{D}$ as $k \rightarrow \infty$. Then by (2.16) and (2.17), it follows that

$$
\begin{aligned}
\| D & C_{\varphi} f_{k} \|_{\mathcal{B}^{\beta}} \\
= & \left(\left|f_{k}^{\prime}(\varphi(0)) \varphi^{\prime}(0)\right|+\sup _{z \in \mathbb{D}}\left(1-|z|^{2}\right)^{\beta}\left|f_{k}^{\prime \prime}(\varphi(z))\left(\varphi^{\prime}(z)\right)^{2}+f_{k}^{\prime}(\varphi(z)) \varphi^{\prime \prime}(z)\right|\right) \\
\leq & \left|f_{k}^{\prime}(\varphi(0)) \varphi^{\prime}(0)\right|+\sup _{z \in \mathbb{D}}\left(1-|z|^{2}\right)^{\beta}\left|f_{k}^{\prime \prime}(\varphi(z))\left(\varphi^{\prime}(z)\right)^{2}\right| \\
& +\sup _{z \in \mathbb{D}}\left(1-|z|^{2}\right)^{\beta}\left|f_{k}^{\prime}(\varphi(z)) \varphi^{\prime \prime}(z)\right| \\
\leq & \left|f_{k}^{\prime}(\varphi(0)) \varphi^{\prime}(0)\right|+\sup _{|w| \leq r}\left|f_{k}^{\prime \prime}(w)\right| \sup _{z \in \mathbb{D}}\left(1-|z|^{2}\right)^{\beta}\left|\varphi^{\prime}(z)\right|^{2} \\
& +\sup _{|w| \leq r}\left|f_{k}^{\prime}(w)\right| \sup _{z \in \mathbb{D}}\left(1-|z|^{2}\right)^{\beta}\left|\varphi^{\prime \prime}(z)\right| \\
\rightarrow & 0, \operatorname{as} k \rightarrow \infty .
\end{aligned}
$$

That is,

$$
\lim _{k \rightarrow \infty}\left\|D C_{\varphi} f_{k}\right\|_{\mathcal{B}^{\beta}}=0 .
$$

From Lemma 6, it is clear that the operator $D C_{\varphi}: \mathcal{B}^{\alpha} \rightarrow \mathcal{B}^{\beta}$ is compact. That is

$$
\left\|D C_{\varphi}\right\|_{e}=0 \text {. }
$$

On the other hand, by (2.16), and since $\lim _{n \rightarrow \infty} n^{\alpha} r^{n-1}=0$, it follows that

$$
\begin{aligned}
A: & =\left(\frac{e}{2(\alpha+1)}\right)^{\alpha+1} \limsup _{n \rightarrow \infty} n^{\alpha}\left\|I_{\varphi^{\prime}}\left(\varphi^{n}\right)\right\|_{\beta} \\
& =\left(\frac{e}{2(\alpha+1)}\right)^{\alpha+1} \limsup _{n \rightarrow \infty} \sup _{z \in \mathbb{D}} n^{\alpha}\left(1-|z|^{2}\right)^{\beta}\left|\varphi^{\prime}(z)\right|^{2}|\varphi(z)|^{n-1} \\
& \leq\left(\frac{e}{2(\alpha+1)}\right)^{\alpha+1} \limsup _{n \rightarrow \infty} n^{\alpha} r^{n-1} \sup _{z \in \mathbb{D}}\left(1-|z|^{2}\right)^{\beta}\left|\varphi^{\prime}(z)\right|^{2} \\
& =0 .
\end{aligned}
$$


Similarly, by (2.17) it follows that

$$
\begin{aligned}
B: & =\left(\frac{e}{2 \alpha}\right)^{\alpha} \limsup _{n \rightarrow \infty} n^{\alpha}\left\|J_{\varphi^{\prime}}\left(\varphi^{n-1}\right)\right\|_{\beta} \\
& =\left(\frac{e}{2 \alpha}\right)^{\alpha} \limsup _{n \rightarrow \infty} n^{\alpha} \sup _{z \in \mathbb{D}}\left(1-|z|^{2}\right)^{\beta}|\varphi(z)|^{n-1}\left|\varphi^{\prime \prime}(z)\right| \\
& \leq\left(\frac{e}{2 \alpha}\right)^{\alpha} \limsup _{n \rightarrow \infty} n^{\alpha} r^{n-1} \sup _{z \in \mathbb{D}}\left(1-|z|^{2}\right)^{\beta}\left|\varphi^{\prime \prime}(z)\right| \\
& =0
\end{aligned}
$$

From (3.2), (3.3) and (3.4), we know that both sides of (3.1) are zero. Thus for the case $\sup _{z \in \mathbb{D}}|\varphi(z)|<1$, our essential norm formula (3.1) is trivially true.

Therefore, in the following, we assume that

$$
\sup _{z \in \mathbb{D}}|\varphi(z)|=1
$$

We first prove the upper bounded. Let $L_{n}$ be the sequence of operators given in Lemmas 3, 4, and 5. Since each $L_{n}$ is compact as an operator from $\mathcal{B}^{\alpha}$ to $\mathcal{B}^{\alpha}$, and $D C_{\varphi} L_{n}: \mathcal{B}^{\alpha} \rightarrow \mathcal{B}^{\beta}$ is also compact. Thus we have that

$$
\begin{aligned}
& \left\|D C_{\varphi}\right\|_{e} \leq \limsup _{n \rightarrow \infty}\left\|D C_{\varphi}-D C_{\varphi} L_{n}\right\| \\
& =\limsup _{n \rightarrow \infty}\left\|D C_{\varphi}\left(I-L_{n}\right)\right\| \\
& =\limsup _{n \rightarrow \infty} \sup _{\|f\|_{\mathcal{B}^{\alpha} \leq 1}} \|\left(D C_{\varphi}\left(I-L_{n}\right) f \|_{\mathcal{B}^{\beta}}\right. \\
& \leq \limsup _{n \rightarrow \infty} \sup _{\|f\|_{\mathcal{B}^{\alpha} \leq 1}}\left|\left(\left(I-L_{n}\right) f\right)^{\prime}(\varphi(0))\right|\left|\varphi^{\prime}(0)\right| \\
& +\limsup _{n \rightarrow \infty} \sup _{\|f\|_{\mathcal{B}^{\alpha} \leq 1}} \sup _{z \in \mathbb{D}}\left|\left(\left(I-L_{n}\right) f\right)^{\prime \prime}(\varphi(z))\right|\left|\varphi^{\prime}(z)\right|^{2}\left(1-|z|^{2}\right)^{\beta} \\
& +\limsup _{n \rightarrow \infty} \sup _{\|f\|_{\mathcal{B}^{\alpha} \leq 1}} \sup _{z \in \mathbb{D}}\left|\left(\left(I-L_{n}\right) f\right)^{\prime}(\varphi(z))\right|\left|\varphi^{\prime \prime}(z)\right|\left(1-|z|^{2}\right)^{\beta} .
\end{aligned}
$$

By Lemma 3 (ii), Lemma 4 (iib) , Lemma 5 (ii) and Cauchy' integral formula, we can obtain that

$$
\limsup _{n \rightarrow \infty} \sup _{\|f\|_{\mathcal{B}^{\alpha} \leq 1}}\left|\left(\left(I-L_{n}\right) f\right)^{\prime}(\varphi(0))\right|\left|\varphi^{\prime}(0)\right|=0 .
$$

Next we consider the term in (3.6)

$$
I:=\sup _{\|f\|_{\mathcal{B}^{\alpha} \leq 1}} \sup _{z \in \mathbb{D}}\left|\left(\left(I-L_{n}\right) f\right)^{\prime \prime}(\varphi(z)) \| \varphi^{\prime}(z)\right|^{2}\left(1-|z|^{2}\right)^{\beta} .
$$

For any positive integer $n \geq 1$, let

$$
D_{n}=\left\{z \in \mathbb{D}: r_{n} \leq|\varphi(z)| \leq r_{n+1}\right\},
$$

where $r_{n}$ is given by (2.3). Let $k$ be the the smallest positive integer such that $D_{k} \neq \emptyset$. Since $\sup _{z \in \mathbb{D}}|\varphi(z)|=1, D_{n}$ is not empty for every integer $n \geq k$, and 
$\mathbb{D}=\bigcup_{n=k}^{\infty} D_{n}$. We divide $I$ into two parts:

$$
\begin{aligned}
& I_{1}:=\sup _{\|f\|_{\mathcal{B}^{\alpha} \leq 1}} \sup _{k \leq i \leq N-1} \sup _{z \in D_{i}}\left|\left(\left(I-L_{n}\right) f\right)^{\prime \prime}(\varphi(z)) \| \varphi^{\prime}(z)\right|^{2}\left(1-|z|^{2}\right)^{\beta}, \\
& I_{2}:=\sup _{\|f\|_{\mathcal{B}^{\alpha} \leq 1}} \sup _{N \leq i} \sup _{z \in D_{i}}\left|\left(\left(I-L_{n}\right) f\right)^{\prime \prime}(\varphi(z)) \| \varphi^{\prime}(z)\right|^{2}\left(1-|z|^{2}\right)^{\beta},
\end{aligned}
$$

where $N$ is a positive integer chosen as follows.

Consider the term $I_{1}$, by (i) of Lemmas 3, 4, 5, Cauchy's integral formula and

$$
\begin{aligned}
& \limsup _{n \rightarrow \infty} I_{1} \\
& =\limsup _{n \rightarrow \infty} \sup _{\|f\|_{\mathcal{B}^{\alpha} \leq 1}} \sup _{k \leq i \leq N-1} \sup _{z \in D_{i}}\left|\left(\left(I-L_{n}\right) f\right)^{\prime \prime}(\varphi(z))\right|\left|\varphi^{\prime}(z)\right|^{2}\left(1-|z|^{2}\right)^{\beta} \\
& \leq \sup _{z \in \mathbb{D}}\left(1-|z|^{2}\right)^{\beta}\left|\varphi^{\prime}(z)\right|^{2} \limsup _{n \rightarrow \infty} \sup _{\|f\|_{\mathcal{B}} \leq 1} \sup _{r_{k} \leq|\varphi(z)| \leq r_{N-1}}\left|\left(\left(I-L_{n}\right) f\right)^{\prime \prime}(\varphi(z))\right| \\
& =0 \text {. }
\end{aligned}
$$

Next consider the term $I_{2}$,

$$
\begin{aligned}
& I_{2}:=\sup _{\|f\|_{\mathcal{B}^{\alpha}} \leq 1} \sup _{N \leq i} \sup _{z \in D_{i}}\left|\left(\left(I-L_{n}\right) f\right)^{\prime \prime}(\varphi(z)) \| \varphi^{\prime}(z)\right|^{2}\left(1-|z|^{2}\right)^{\beta} \\
& =\sup _{\|f\|_{\mathcal{B}^{\alpha} \leq 1}} \sup _{N \leq i} \sup _{z \in D_{i}} \frac{\left|\left(\left(I-L_{n}\right) f\right)^{\prime \prime}(\varphi(z)) \| \varphi^{\prime}(z)\right|^{2}\left(1-|z|^{2}\right)^{\beta}|\varphi(z)|^{i-1}\left(1-|\varphi(z)|^{2}\right)^{\alpha+1} i^{\alpha+1}}{|\varphi(z)|^{i-1}\left(1-|\varphi(z)|^{2}\right)^{\alpha+1} i^{\alpha+1}} .
\end{aligned}
$$

Since

$$
|\varphi(z)|^{i-1}\left(1-|\varphi(z)|^{2}\right)^{\alpha+1} i^{\alpha+1} \geq i^{\alpha+1} \min _{x \in\left[r_{i}, r_{i+1}\right]} H_{n, \alpha+1}(x),
$$

and by Lemma 1, we have that

$$
\lim _{i \rightarrow \infty} i^{\alpha+1} \min _{x \in\left[r_{i}, r_{i+1}\right]} H_{n, \alpha+1}(x)=\left(\frac{2(\alpha+1)}{e}\right)^{\alpha+1} .
$$

Therefore, for an arbitrary $\varepsilon>0$, we can find $N>k+1$, and $N$ large enough such that for any $i \geq N$,

$$
\left[|\varphi(z)|^{i-1}\left(1-|\varphi(z)|^{2}\right)^{\alpha+1} i^{\alpha+1}\right]^{-1}<\left(\frac{e}{2(\alpha+1)}\right)^{\alpha+1}+\varepsilon
$$

Then by the above inequality and (2.6), we have that

$$
\begin{aligned}
& \limsup _{n \rightarrow \infty} I_{2} \leq\left(\left(\frac{e}{2(\alpha+1)}\right)^{\alpha+1}+\varepsilon\right)
\end{aligned}
$$

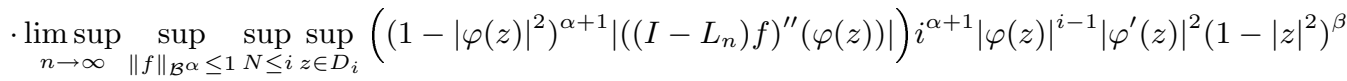

$$
\begin{aligned}
& \preceq\left(\left(\frac{e}{2(\alpha+1)}\right)^{\alpha+1}+\varepsilon\right) \sup _{\|f\|_{\mathcal{B}^{\alpha} \leq 1}}\left\|\left(I-L_{n}\right) f\right\|_{\mathcal{B}^{\alpha}} \sup _{N \leq i} \sup _{z \in D_{i}} i^{\alpha} i\left|\varphi^{\prime}(z)\right|^{2}|\varphi(z)|^{i-1}\left(1-|z|^{2}\right)^{\beta} \\
& \preceq\left(\left(\frac{e}{2(\alpha+1)}\right)^{\alpha+1}+\varepsilon\right)\left\|I-L_{n}\right\| \sup _{N \leq i} i^{\alpha}\left\|I_{\varphi^{\prime}}\left(\varphi^{i}\right)\right\|_{\beta} \\
& \preceq\left(\left(\frac{e}{2(\alpha+1)}\right)^{\alpha+1}+\varepsilon\right) \sup _{N \leq i} i^{\alpha}\left\|I_{\varphi^{\prime}}\left(\varphi^{i}\right)\right\|_{\beta} .
\end{aligned}
$$

Where the last inequality follows from the (iii) of Lemmas 3, 4, and 5 . 
From (3.10) and (3.11), it follows that the term in (3.6) is less than

$$
C\left(\left(\frac{e}{2(\alpha+1)}\right)^{\alpha+1}+\varepsilon\right) \sup _{N \leq i} i^{\alpha}\left\|I_{\varphi^{\prime}}\left(\varphi^{i}\right)\right\|_{\beta} .
$$

Employing the similar argument as that of (3.6), we get the term in (3.7) is less than

$$
C\left(\left(\frac{e}{2 \alpha}\right)^{\alpha}+\varepsilon\right) \sup _{N \leq i} i^{\alpha}\left\|J_{\varphi^{\prime}}\left(\varphi^{i-1}\right)\right\|_{\beta}
$$

where $\varepsilon$ and $N$ are given as above ( $\mathrm{N}$ may be not the same, we can choose the bigger one). Hence

$$
\begin{aligned}
\left\|D C_{\varphi}\right\|_{e} \preceq & \left(\left(\frac{e}{2(\alpha+1)}\right)^{\alpha+1}+\varepsilon\right) \sup _{N \leq i} i^{\alpha}\left\|I_{\varphi^{\prime}}\left(\varphi^{i}\right)\right\|_{\beta} \\
+ & \left(\left(\frac{e}{2 \alpha}\right)^{\alpha}+\varepsilon\right) \sup _{N \leq i} i^{\alpha}\left\|J_{\varphi^{\prime}}\left(\varphi^{i-1}\right)\right\|_{\beta} .
\end{aligned}
$$

Since $\varepsilon$ is an arbitrary positive number, we obtain that

$$
\begin{aligned}
\left\|D C_{\varphi}\right\|_{e} & \preceq\left(\frac{e}{2(\alpha+1)}\right)^{\alpha+1} \limsup _{i \rightarrow \infty} i^{\alpha}\left\|I_{\varphi^{\prime}}\left(\varphi^{i}\right)\right\|_{\beta}+\left(\frac{e}{2 \alpha}\right)^{\alpha} \limsup _{i \rightarrow \infty} i^{\alpha}\left\|J_{\varphi^{\prime}}\left(\varphi^{i-1}\right)\right\|_{\beta} \\
& \preceq A+B .
\end{aligned}
$$

That is the upper estimate.

Now, we still suppose that $\sup _{z \in \mathbb{D}}|\varphi(z)|=1$. We prove the lower estimate. Take every compact operator $K: \mathcal{B}^{\alpha} \rightarrow \mathcal{B}^{\beta}$. Then for each sequence $\left\{f_{k}\right\}_{k \in \mathbb{N}}$ in $\mathcal{B}^{\alpha}$ with $\left\|f_{k}\right\|_{\mathcal{B}^{\alpha}} \leq 1$, and $f_{k}$ converges to zero weakly in $\mathcal{B}^{\alpha}$, it follows that

$$
\lim _{k \rightarrow \infty}\left\|K f_{k}\right\|_{\mathcal{B}^{\beta}}=0
$$

Therefore,

$$
\left\|D C_{\varphi}-K\right\| \geq \limsup _{k \rightarrow \infty}\left\|\left(D C_{\varphi}-K\right) f_{k}\right\|_{\mathcal{B}^{\beta}} \geq \limsup _{k \rightarrow \infty}\left\|D C_{\varphi}\left(f_{k}\right)\right\|_{\mathcal{B}^{\beta}}
$$

Choose a sequence $\left\{a_{k}\right\}_{k \in \mathbb{N}}$ in $\mathbb{D}$ such that $\left|\varphi\left(a_{k}\right)\right| \rightarrow 1$ as $k \rightarrow \infty$. For any $\alpha>0$ consider the functions $f_{k}$ defined by

$$
f_{k}(z)=(\alpha+1) \frac{1-\left|\varphi\left(a_{k}\right)\right|^{2}}{\left(1-\overline{\varphi\left(a_{k}\right)} z\right)^{\alpha}}-\alpha \frac{\left(1-\left|\varphi\left(a_{k}\right)\right|^{2}\right)^{2}}{\left(1-\overline{\varphi\left(a_{k}\right)} z\right)^{\alpha+1}} .
$$

Clearly $f_{k}(z)$ converges to zero uniformly on compact subsets of $\mathbb{D}$. Notice that

$$
f_{k}^{\prime}(z)=\alpha(\alpha+1) \overline{\varphi\left(a_{k}\right)} \frac{1-\left|\varphi\left(a_{k}\right)\right|^{2}}{\left(1-\overline{\varphi\left(a_{k}\right)} z\right)^{\alpha+1}}-\alpha(\alpha+1) \overline{\varphi\left(a_{k}\right)} \frac{\left(1-\left|\varphi\left(a_{k}\right)\right|^{2}\right)^{2}}{\left(1-\overline{\varphi\left(a_{k}\right)} z\right)^{\alpha+2}}
$$

and

$$
\begin{aligned}
f_{k}^{\prime \prime}(z)= & \alpha(\alpha+1)^{2}\left(\overline{\varphi\left(a_{k}\right)}\right)^{2} \frac{1-\left|\varphi\left(a_{k}\right)\right|^{2}}{\left(1-\overline{\varphi\left(a_{k}\right)} z\right)^{\alpha+2}} \\
& -\alpha(\alpha+1)(\alpha+2)\left(\overline{\varphi\left(a_{k}\right)}\right)^{2} \frac{\left(1-\left|\varphi\left(a_{k}\right)\right|^{2}\right)^{2}}{\left(1-\overline{\varphi\left(a_{k}\right)} z\right)^{\alpha+3}}
\end{aligned}
$$


Thus it follows that

$$
f_{k}^{\prime}\left(\varphi\left(a_{k}\right)\right)=0, \quad f_{k}^{\prime \prime}\left(\varphi\left(a_{k}\right)\right)=-\frac{\alpha(\alpha+1)\left(\overline{\varphi\left(a_{k}\right)}\right)^{2}}{\left(1-\left|\varphi\left(a_{k}\right)\right|^{2}\right)^{\alpha+1}}
$$

and

$$
\begin{aligned}
\left|f_{k}^{\prime}(z)\right| & \leq \alpha(\alpha+1) \frac{1-\left|\varphi\left(a_{k}\right)\right|^{2}}{(1-|z|)^{\alpha}\left(1-\left|\varphi\left(a_{k}\right)\right|\right)}+\alpha(\alpha+1) \frac{\left(1-\left|\varphi\left(a_{k}\right)\right|^{2}\right)^{2}}{(1-|z|)^{\alpha}\left(1-\left|\varphi\left(a_{k}\right)\right|\right)^{2}} \\
& \leq \alpha(\alpha+1) \frac{2^{\alpha+1}+2^{\alpha+2}}{\left(1-|z|^{2}\right)^{\alpha}}=\frac{3 \alpha(\alpha+1) 2^{\alpha+1}}{\left(1-|z|^{2}\right)^{\alpha}} .
\end{aligned}
$$

From which it follows that

$$
\left\|f_{k}\right\|_{\mathcal{B}^{\alpha}} \leq\left|f_{k}(0)\right|+3 \alpha(\alpha+1) 2^{\alpha+1} .
$$

Let $\widetilde{f_{k}(z)}=f_{k}(z) /\left\|f_{k}\right\|_{\mathcal{B}^{\alpha}}$. Then $\left\|\widetilde{f}_{k}\right\|_{\mathcal{B}^{\alpha}}=1$, and $\widetilde{f}_{k}$ converges to zero uniformly on compact subsets of $\mathbb{D}$. Then by (3.12) and (3.13), it follows that

$$
\begin{aligned}
\left\|D C_{\varphi}\right\|_{e} & \geq \limsup _{k \rightarrow \infty}\left\|D C_{\varphi}\left(\tilde{f}_{k}\right)\right\|_{\mathcal{B}^{\beta}} \\
& \geq \limsup _{k \rightarrow \infty}\left\|D C_{\varphi}\left(\tilde{f}_{k}\right)\right\|_{\beta} \\
& =\limsup _{k \rightarrow \infty} \frac{\left\|D C_{\varphi}\left(f_{k}\right)\right\|_{\beta}}{\left\|f_{k}\right\|_{\mathcal{B}^{\alpha}}} \\
& \geq \limsup _{k \rightarrow \infty} \frac{\left(1-\left|a_{k}\right|^{2}\right)^{\beta}\left|f^{\prime \prime}\left(\varphi\left(a_{k}\right)\right)\left(\varphi^{\prime}\left(a_{k}\right)\right)^{2}+f^{\prime}\left(\varphi\left(a_{k}\right)\right) \varphi^{\prime \prime}\left(a_{k}\right)\right|}{\left|f_{k}(0)\right|+3 \alpha(\alpha+1) 2^{\alpha+1}} \\
& =\limsup _{k \rightarrow \infty} \frac{\left(1-\left|a_{k}\right|^{2}\right)^{\beta}\left|f^{\prime \prime}\left(\varphi\left(a_{k}\right)\right)\left(\varphi^{\prime}\left(a_{k}\right)\right)^{2}\right|}{\left|f_{k}(0)\right|+3 \alpha(\alpha+1) 2^{\alpha+1}} \\
& =\limsup _{k \rightarrow \infty} \frac{1}{\left|f_{k}(0)\right|+3 \alpha(\alpha+1) 2^{\alpha+1}} \frac{1}{1}\left(1-\left|a_{k}\right|^{2}\right)^{\beta}\left|\varphi^{\prime}\left(a_{k}\right)\right|^{2} \\
& =\limsup _{k \rightarrow \infty} \frac{\left(\left.a_{k}\right|^{2}\right)^{\beta}\left|\varphi^{\prime}\left(a_{k}\right)\right|^{2}\left|\overline{\varphi\left(a_{k}\right)}\right|^{2}}{\left|f_{k}(0)\right|+3 \cdot 2^{\alpha+1}}
\end{aligned}
$$

Since

$$
\lim _{n \rightarrow \infty}\left|f_{k}(0)\right|=\lim _{k \rightarrow \infty}\left((\alpha+1)\left(1-\left|\varphi\left(a_{k}\right)\right|^{2}\right)-\alpha\left(1-\left|\varphi\left(a_{k}\right)\right|^{2}\right)^{2}\right)=0,
$$

we obtain that

$$
\left\|D C_{\varphi}\right\|_{e} \geq \frac{1}{3 \cdot 2^{\alpha+1}} \lim _{|\varphi(z)| \rightarrow 1} \frac{\left(1-|z|^{2}\right)^{\beta}\left|\varphi^{\prime}(z)\right|^{2}}{\left(1-|\varphi(z)|^{2}\right)^{\alpha+1}} .
$$

On the other hand, we consider the following term

$$
\begin{aligned}
& \left(\frac{e}{2(\alpha+1)}\right)^{\alpha+1} n^{\alpha}\left\|I_{\varphi^{\prime}}\left(\varphi^{n}\right)\right\|_{\beta} \\
= & \left(\frac{e}{2(\alpha+1)}\right)^{\alpha+1} n^{\alpha+1} \sup _{z \in \mathbb{D}}\left(1-|z|^{2}\right)^{\beta}|\varphi(z)|^{n-1}\left|\varphi^{\prime}(z)\right|^{2} \\
= & L_{1}+L_{2} .
\end{aligned}
$$


Where

$$
\begin{aligned}
L_{1} & :=\left(\frac{e}{2(\alpha+1)}\right)^{\alpha+1} n^{\alpha+1} \sup _{|\varphi(z)| \leq s}\left(1-|z|^{2}\right)^{\beta}|\varphi(z)|^{n-1}\left|\varphi^{\prime}(z)\right|^{2}, \\
L_{2} & :=\left(\frac{e}{2(\alpha+1)}\right)^{\alpha+1} n^{\alpha+1} \sup _{|\varphi(z)|>s}\left(1-|z|^{2}\right)^{\beta}|\varphi(z)|^{n-1}\left|\varphi^{\prime}(z)\right|^{2} .
\end{aligned}
$$

here $s \in(0,1)$. For $L_{1}$, by (2.16) it follows that

$$
\begin{aligned}
L_{1}: & =\left(\frac{e}{2(\alpha+1)}\right)^{\alpha+1} n^{\alpha+1} \sup _{|\varphi(z)| \leq s}\left(1-|z|^{2}\right)^{\beta}|\varphi(z)|^{n-1}\left|\varphi^{\prime}(z)\right|^{2} \\
& \leq\left(\frac{e}{2(\alpha+1)}\right)^{\alpha+1}\left(\sup _{z \in \mathbb{D}}\left(1-|z|^{2}\right)^{\beta}\left|\varphi^{\prime}(z)\right|^{2}\right) n^{\alpha+1} s^{n-1} .
\end{aligned}
$$

Thus

$$
\limsup _{n \rightarrow \infty} L_{1}=0 \text {. }
$$

For $L_{2}$, by Lemma 1, it is clear that

$$
\begin{aligned}
L_{2}: & =\left(\frac{e}{2(\alpha+1)}\right)^{\alpha+1} n^{\alpha+1} \sup _{|\varphi(z)|>s}\left(1-|z|^{2}\right)^{\beta}|\varphi(z)|^{n-1}\left|\varphi^{\prime}(z)\right|^{2} \\
& =\left(\frac{e}{2(\alpha+1)}\right)^{\alpha+1} \sup _{|\varphi(z)|>s} \frac{\left(1-|z|^{2}\right)^{\beta}\left|\varphi^{\prime}(z)\right|^{2} n^{\alpha+1}\left(|\varphi(z)|^{n-1}\left(1-|\varphi(z)|^{2}\right)^{\alpha+1}\right)}{\left(1-|\varphi(z)|^{2}\right)^{\alpha+1}} \\
& \leq\left(\frac{e}{2(\alpha+1)}\right)^{\alpha+1} n^{\alpha+1} H_{n, \alpha+1}\left(r_{n}\right) \sup _{|\varphi(z)|>s} \frac{\left(1-|z|^{2}\right)^{\beta}\left|\varphi^{\prime}(z)\right|^{2}}{\left(1-|\varphi(z)|^{2}\right)^{\alpha+1}}
\end{aligned}
$$

where $r_{n}$ is defined in (2.3). Since

$$
\lim _{n \rightarrow \infty} n^{\alpha+1} H_{n, \alpha+1}\left(r_{n}\right)=\left(\frac{2(\alpha+1)}{e}\right)^{\alpha+1},
$$

we have that

$$
\begin{aligned}
\limsup _{n \rightarrow \infty} L_{2} & \leq \limsup _{n \rightarrow \infty}\left(\frac{e}{2(\alpha+1)}\right)^{\alpha+1} n^{\alpha+1} H_{n, \alpha+1}\left(r_{n}\right) \sup _{|\varphi(z)|>s} \frac{\left(1-|z|^{2}\right)^{\beta}\left|\varphi^{\prime}(z)\right|^{2}}{\left(1-|\varphi(z)|^{2}\right)^{\alpha+1}} \\
& =\left(\frac{e}{2(\alpha+1)}\right)^{\alpha+1}\left(\frac{2(\alpha+1)}{e}\right)^{\alpha+1} \sup _{|\varphi(z)|>s} \frac{\left(1-|z|^{2}\right)^{\beta}\left|\varphi^{\prime}(z)\right|^{2}}{\left(1-|\varphi(z)|^{2}\right)^{\alpha+1}} \\
& =\sup _{|\varphi(z)|>s} \frac{\left(1-|z|^{2}\right)^{\beta}\left|\varphi^{\prime}(z)\right|^{2}}{\left(1-|\varphi(z)|^{2}\right)^{\alpha+1}} .
\end{aligned}
$$

Therefore,

$$
\begin{aligned}
& \limsup _{n \rightarrow \infty}\left(\frac{e}{2(\alpha+1)}\right)^{\alpha+1} n^{\alpha}\left\|I_{\varphi^{\prime}}\left(\varphi^{n}\right)\right\|_{\beta} \\
& \leq \lim _{s \rightarrow 1} \sup _{|\varphi(z)|>s} \frac{\left(1-|z|^{2}\right)^{\beta}\left|\varphi^{\prime}(z)\right|^{2}}{\left(1-|\varphi(z)|^{2}\right)^{\alpha+1}} \\
& \leq \limsup _{|\varphi(z)| \rightarrow 1} \frac{\left(1-|z|^{2}\right)^{\beta}\left|\varphi^{\prime}(z)\right|^{2}}{\left(1-|\varphi(z)|^{2}\right)^{\alpha+1}}
\end{aligned}
$$


From (3.15) and (3.14), it follows that

$$
\begin{aligned}
\left\|D C_{\varphi}\right\|_{e} & \geq \frac{1}{3 \cdot 2^{\alpha+1}}\left(\frac{e}{2(\alpha+1)}\right)^{\alpha+1} \limsup _{n \rightarrow \infty} n^{\alpha}\left\|I_{\varphi^{\prime}}\left(\varphi^{n}\right)\right\|_{\beta} \\
& =\frac{A}{3 \cdot 2^{\alpha+1}} .
\end{aligned}
$$

Next we proceed to prove the other lower bound in a similar way as above. We still choose a sequence $\left\{a_{k}\right\}_{k \in \mathbb{N}}$ in $\mathbb{D}$ such that $\left|\varphi\left(a_{k}\right)\right| \rightarrow 1$ as $k \rightarrow \infty$. For any $\alpha>0$ consider the functions $g_{k}$ defined by

$$
g_{k}(z)=(\alpha+2) \frac{1-\left|\varphi\left(a_{k}\right)\right|^{2}}{\left(1-\overline{\varphi\left(a_{k}\right)} z\right)^{\alpha}}-\alpha \frac{\left(1-\left|\varphi\left(a_{k}\right)\right|^{2}\right)^{2}}{\left(1-\overline{\varphi\left(a_{k}\right)} z\right)^{\alpha+1}}
$$

Clearly $g_{k}(z)$ converges to zero uniformly on compact subsets of $\mathbb{D}$. Moreover,

$$
\begin{gathered}
g_{k}^{\prime}(z)=\alpha(\alpha+2) \frac{\left(1-\left|\varphi\left(a_{k}\right)\right|^{2}\right) \overline{\varphi\left(a_{k}\right)}}{\left(1-\overline{\varphi\left(a_{k}\right)} z\right)^{\alpha+1}}-\alpha(\alpha+1) \frac{\left(1-\left|\varphi\left(a_{k}\right)\right|^{2}\right)^{2} \overline{\varphi\left(a_{k}\right)}}{\left(1-\overline{\varphi\left(a_{k}\right)} z\right)^{\alpha+2}} \\
g_{k}^{\prime \prime}(z)=\alpha(\alpha+1)(\alpha+2) \frac{\left(1-\left|\varphi\left(a_{k}\right)\right|^{2}\right)\left(\overline{\varphi\left(a_{k}\right)}\right)^{2}}{\left(1-\overline{\varphi\left(a_{k}\right)} z\right)^{\alpha+2}} \\
-\alpha(\alpha+1)(\alpha+2) \frac{\left(1-\left|\varphi\left(a_{k}\right)\right|^{2}\right)^{2}\left(\overline{\varphi\left(a_{k}\right)}\right)^{2}}{\left(1-\overline{\varphi\left(a_{k}\right)} z\right)^{\alpha+3}} \\
g_{k}^{\prime}\left(\varphi\left(a_{k}\right)\right)=\frac{\alpha \overline{\varphi\left(a_{k}\right)}}{\left(1-\left|\varphi\left(a_{k}\right)\right|^{2}\right)^{\alpha}}, \quad g_{k}^{\prime \prime}\left(\varphi\left(a_{k}\right)\right)=0 .
\end{gathered}
$$

and

$$
\begin{aligned}
\left|g_{k}^{\prime}(z)\right| & \leq \alpha(\alpha+2) \frac{1-\left|\varphi\left(a_{k}\right)\right|^{2}}{\left(1-\left|\varphi\left(a_{k}\right)\right|\right)(1-|z|)^{\alpha}}+\alpha(\alpha+1) \frac{\left(1-\left|\varphi\left(a_{k}\right)\right|^{2}\right)^{2}}{\left(1-\left|\varphi\left(a_{k}\right)\right|\right)^{2}(1-|z|)^{\alpha}} \\
& \leq \frac{\alpha(\alpha+2) 2^{\alpha+1}+\alpha(\alpha+1) 2^{\alpha+2}}{\left(1-|z|^{2}\right)^{\alpha}} \\
& =\frac{2^{\alpha+1} \alpha(3 \alpha+4)}{\left(1-|z|^{2}\right)^{\alpha}} .
\end{aligned}
$$

Besides,

$$
g_{k}(0)=(\alpha+2)\left(1-\left|\varphi\left(a_{k}\right)\right|^{2}\right)-\alpha\left(1-\left|\varphi\left(a_{k}\right)\right|^{2}\right)^{2} \rightarrow 0, k \rightarrow \infty
$$


We choose the test functions $\widetilde{g_{k}(z)}=g_{k} /\left\|g_{k}\right\|_{\mathcal{B}^{\alpha}}$. Then it follows that

$$
\begin{aligned}
\left\|D C_{\varphi}\right\|_{e} & \geq \limsup _{k \rightarrow \infty}\left\|D C_{\varphi}\left(\widetilde{g_{k}}\right)\right\|_{\mathcal{B}^{\beta}} \\
& \geq \limsup _{k \rightarrow \infty}\left\|D C_{\varphi}\left(\widetilde{g_{k}}\right)\right\|_{\beta} \\
& =\limsup _{k \rightarrow \infty} \frac{\left\|D C_{\varphi}\left(g_{k}\right)\right\|_{\beta}}{\left\|g_{k}\right\|_{\mathcal{B}^{\alpha}}} \\
& \geq \limsup _{k \rightarrow \infty} \frac{\left(1-\left|a_{k}\right|^{2}\right)^{\beta}\left|g^{\prime \prime}\left(\varphi\left(a_{k}\right)\right)\left(\varphi^{\prime}\left(a_{k}\right)\right)^{2}+g^{\prime}\left(\varphi\left(a_{k}\right)\right) \varphi^{\prime \prime}\left(a_{k}\right)\right|}{\left|g_{k}(0)\right|+2^{\alpha+1} \alpha(3 \alpha+4)} \\
& =\limsup _{k \rightarrow \infty} \frac{\left(1-\left|a_{k}\right|^{2}\right)^{\beta}\left|g^{\prime}\left(\varphi\left(a_{k}\right)\right) \varphi^{\prime \prime}\left(a_{k}\right)\right|}{\left|g_{k}(0)\right|+2^{\alpha+1} \alpha(3 \alpha+4)} \\
& =\limsup _{k \rightarrow \infty} \frac{1}{2^{\alpha+1} \alpha(3 \alpha+4)} \frac{\alpha\left(1-\left|a_{k}\right|^{2}\right)^{\beta}\left|\overline{\varphi\left(a_{k}\right)}\right|\left|\varphi^{\prime \prime}\left(a_{k}\right)\right|}{\left(1-\left|\varphi\left(a_{k}\right)\right|^{2}\right)^{\alpha}} \\
& =\frac{1}{2^{\alpha+1}(3 \alpha+4)} \limsup _{|\varphi(z)| \rightarrow 1} \frac{\left(1-|z|^{2}\right)^{\beta}\left|\varphi^{\prime \prime}(z)\right|}{\left(1-|\varphi(z)|^{2}\right)^{\alpha}} .
\end{aligned}
$$

On the other hand, using the similar proof as that of (3.15), we have that

$$
\begin{aligned}
& \left(\frac{e}{2 \alpha}\right)^{\alpha} n^{\alpha}\left\|J_{\varphi^{\prime}}\left(\varphi^{n-1}\right)\right\|_{\beta} \\
& \leq \lim _{s \rightarrow 1} \sup _{|\varphi(z)|>s} \frac{\left(1-|z|^{2}\right)^{\beta}\left|\varphi^{\prime \prime}(z)\right|}{\left(1-|\varphi(z)|^{2}\right)^{\alpha}} \\
& \leq \limsup _{|\varphi(z)| \rightarrow 1} \frac{\left(1-|z|^{2}\right)^{\beta}\left|\varphi^{\prime \prime}(z)\right|}{\left(1-|\varphi(z)|^{2}\right)^{\alpha}} .
\end{aligned}
$$

Then it is clear that

$$
\begin{aligned}
\left\|D C_{\varphi}\right\|_{e} & \geq \frac{1}{2^{\alpha+1}(3 \alpha+4)} \limsup _{|\varphi(z)| \rightarrow 1} \frac{\left(1-|z|^{2}\right)^{\beta}\left|\varphi^{\prime \prime}(z)\right|}{\left(1-|\varphi(z)|^{2}\right)^{\alpha}} \\
& =\frac{B}{2^{\alpha+1}(3 \alpha+4)} .
\end{aligned}
$$

From (3.16) and (3.17) we obtain the lower bound for the essential norm. This completes the proof.

The following result is an immediate consequence of Theorem 2.

Corollary 3.6. Let $0<\alpha, \beta<\infty$ and $\varphi \in S(\mathbb{D})$. Suppose that the operator $D C_{\varphi}: \mathcal{B}^{\alpha} \rightarrow \mathcal{B}^{\beta}$ is bounded. Then $D C_{\varphi}: \mathcal{B}^{\alpha} \rightarrow \mathcal{B}^{\beta}$ is compact if and only if

$$
\limsup _{n \rightarrow \infty} n^{\alpha}\left\|I_{\varphi^{\prime}}\left(\varphi^{n}\right)\right\|_{\beta}=0
$$

and

$$
\limsup _{n \rightarrow \infty} n^{\alpha}\left\|J_{\varphi^{\prime}}\left(\varphi^{n-1}\right)\right\|_{\beta}=0 .
$$

Combing Theorem D with our Corollary 2, it is easy to obtain Corollary 3 below. 
Corollary 3.7. Let $0<\alpha, \beta<\infty$ and $\varphi \in S(\mathbb{D})$. Suppose that the operator $D C_{\varphi}: \mathcal{B}^{\alpha} \rightarrow \mathcal{B}^{\beta}$ is bounded. Then the following statements are equivalent.

(a)

$$
\limsup _{n \rightarrow \infty} n^{\alpha}\left\|I_{\varphi^{\prime}}\left(\varphi^{n}\right)\right\|_{\beta}=0 \quad \text { and } \quad \limsup _{n \rightarrow \infty} n^{\alpha}\left\|J_{\varphi^{\prime}}\left(\varphi^{n-1}\right)\right\|_{\beta}=0 .
$$

$$
\lim _{|\varphi(z)| \rightarrow 1} \frac{\left|\varphi^{\prime}(z)\right|^{2}\left(1-|z|^{2}\right)^{\beta}}{\left(1-|\varphi(z)|^{2}\right)^{\alpha+1}}=0 \quad \text { and } \quad \lim _{|\varphi(z)| \rightarrow 1} \frac{\left|\varphi^{\prime \prime}(z)\right|\left(1-|z|^{2}\right)^{\beta}}{\left(1-|\varphi(z)|^{2}\right)^{\alpha}}=0 .
$$

Acknowledgement. The authors thank the editor and referees for many useful comments and suggestions. This research is supported in part by the National Natural Science Foundation of China (Grant Nos. 11371276,11301373, 11126164, 11201131).

\section{REFERENCES}

1. R. Aron and M. Lindström, Spectra of weighted composition operators on weighted Banach spaces of analytic functions, Israel J. of Math. 141 (2004), 263-276.

2. R.E. Castillo, D.D. Clahane, J.F. Farías López and J.C. Ramos Fernández, Composition operators from logarithmic Bloch spaces to weighted Bloch spaces, Appl. Math. Comp. 219 (2013), 6692-6706.

3. C.C. Cowen and B.D. MacCluer, Composition Operators on Spaces of Analytic Functions, CRC Press, Boca Raton, FL, 1995.

4. Z.S. Fang and Z.H. Zhou, Essential norms of composition operators between Bloch type spaces in the polydisk, Archiv der Mathematik 99 (2012), no. 6, 547-556.

5. P. Gorkin and B.D. MacCluer, Essential norms of composition operators, Integral Equations Operator Theory 48 (2004), 27-40.

6. O. Hyvärinen and M. Lindström, Estimates of essential norms of weighted composition operators between Bloch-type spaces, J. Math. Anal. Appl. 393 (2012), 38-44.

7. O. Hyvärinen, M. Kemppainen, M. Lindström, A. Rautio, and E. Saukko, The essential norm of weighted composition operators on weighted Banach spaces of analytic functions, Integral Equations Operator Theory 72 (2012), 151-157.

8. S. Li and S. Stević, Composition followed by differentiation between Bloch type spaces, J. Comput. Anal. Appl. 9 (2007), no. 2, 195-206.

9. B.D. MacCluer and R. Zhao, Essential norms of weighted composition operators between Bloch-type spaces, Rocky Mountain J. Math. 33 (2003), 1437-1458.

10. J.S. Manhas and R. Zhao, New estimates of essential norms of weighted composition operators between Bloch type spaces, J. Math. Anal. Appl. 389 (2012), 32-47.

11. J.C. Ramos-Fernández, Composition operators on Bloch-Orlicz type spaces, Appl. Math. Comp. 217 (2010), 3392-3402.

12. J.H. Shapiro, Composition operators and Classical Function Theory, Springer-Verlag, New York, 1993.

13. S. Stević, Characterizations of composition followed by differentiation between Bloch-type spaces, Appl. Math. Comput. 218 (2011), 4312-4316.

14. Y. Wu and H. Wulan, Products of differentiation and composition operators on the Bloch space, Collect. Math. 63 (2012), 93-107.

15. H. Wulan, D. Zheng, and K. Zhu, Compact composition operators on BMOA and the Bloch space, Proc. Amer. Math. Soc. 137 (2009), 3861-3868.

16. R. Zhao, Essential norms of composisition operators between Bloch type spces, Proc. Amer. Math. Soc. 138 (2010), 2537-2546.

17. Z.H. Zhou and R.Y. Chen, Weighted composition operators fom $F(p, q, s)$ to Bloch type spaces, Internat. J. Math. 19 (2008), no. 8, 899-926. 
18. Z.H. Zhou and J.H. Shi, Compactness of composition operators on the Bloch space in classical bounded symmetric domains, Michigan Math. J. 50 (2002), 381-405.

19. L. Zhang and Z.H. Zhou, Hilbert-Schmidt differences of composition operators between the weighted Bergman spaces on the unit ball, Banach J. Math. Anal. 7 (2013), 160-172.

20. H.G. Zeng and Z.H. Zhou, Essential norm estimate of a composition operator between Bloch-type spaces in the unit ball, Rocky Mountain J. Math. 42 (2012), 1049-1071.

21. K. Zhu, Operator Theory in Function Spces, Marcel Dekker, New YOrk, 1990.

Department of Mathematics, Tianjin University, Tianjin 300072, P.R. China.

E-mail address: liangyx1986@126.com

E-mail address: zehuazhoumath@aliyun.com; zhzhou@tju.edu.cn 